\title{
Repeated Exposure to "meaningless" Pseudowords Modulates LPC, but Not N(FN)400
}

\author{
Beatriz Bermúdez-Margaretto · David Beltrán · \\ Alberto Domínguez $\cdot$ Fernando Cuetos
}

Received: 25 April 2014/Accepted: 19 September 2014/Published online: 30 September 2014

(C) Springer Science+Business Media New York 2014

\begin{abstract}
Training readers to recognize pseudowords could decrease the processing differences between them and real words while clarifying the lexical acquisition processes. We analyze the effect of pseudoword repetition through the recording of EEG during a lexical decision task. Results showed a functional dissociation between two well-known ERP components: FN400 (Frontal N400, traditionally related to semantic processes) and LPC (Late Positive Complex, related to memory processes). On the one hand, FN400 was unaffected by pseudoword repetition and showed the typical lexicality effect. On the other hand, topographic and neural source analyses showed that LPC amplitude increased across repetitions, causing the lexicality effect to disappear, with the left inferior frontal, left superior temporal and right superior frontal gyri identified as the most likely neural sources. The lack of repetition effect on FN400 suggests that this component is unrelated to familiarity processes and is only influenced by semantic differences between stimuli. The LPC observations, however, reflect the construction and strengthening of visual memory traces for repeated pseudowords, facilitating their processing over the course of the task.
\end{abstract}

B. Bermúdez-Margaretto $(\bowtie) \cdot$ F. Cuetos

Facultad de Psicología, Universidad de Oviedo, Plaza Feijoo, s/n, 33010 Oviedo, Spain

e-mail: bermudezbeatriz@uniovi.es

D. Beltrán

Laboratorio Neurocog, Universidad de La Laguna, Tenerife, Spain

A. Domínguez

Facultad de Psicología, Universidad de La Laguna, Tenerife, Spain
Keywords Reading - ERP methodology $\cdot$ FN400 - LPC · TANCOVA

\section{Introduction}

Pseudowords are invented linguistic stimuli not previously seen by readers. They can be read out correctly, just like unknown real words, by transforming the sublexical units, such as letters or syllables, into sounds (phonological recoding). However, familiar words and pseudowords are clearly processed in different ways; this is known as the lexicality effect. Words are read more quickly and accurately than pseudowords because readers are familiar with, and have semantic knowledge of, words but not of pseudowords. The most probable causes for this lexicality effect are therefore the differences between the two types of stimuli in terms of their familiarity (pseudowords are unfamiliar to readers) and semantic value (pseudowords are meaningless to readers).

Because all words can be considered to be like pseudowords before they are learned, that is, unfamiliar and meaningless, these stimuli could be used to emulate the processes of word acquisition and consolidation. Training readers in these stimuli, either by means of repeated exposure (improving familiarity) or by repeated association with a meaning (providing semantic features), should promote their integration as new entities in the reader's memory, therefore removing the lexicality effect of pseudowords. Some behavioral studies have shown that familiarity and semantic training in pseudowords are associated to effects that indicate their integration into the reader's lexicon. For example, both reductions of the length effect for pseudowords (Maloney et al. 2009) and increases of lexical competition between words and 
pseudowords (Dumay and Gaskell 2007) have been observed after repeated exposure to the latter stimuli. Furthermore, interference effects were found in a picture naming task when pseudowords, previously associated with a picture, were presented embedded in pictures (as distractors) that belonged to the same semantic category as the pictures with which the distractor pseudowords were associated (Claye et al. 2007). Pseudowords previously associated with pictures also yielded priming effects when presented as primes on a categorization task of these pictures (Breitenstein et al. 2007). These effects are only possible if the trained pseudowords had been integrated in the lexicon and the semantic system of readers, thus causing interference or facilitation on processing of other words with lexical representation.

Studies using ERP methodology have found a signature pattern of brain activity for the lexicality effect. N400, a negative component which peaks around $400 \mathrm{~ms}$, with a posterior scalp distribution, is the ERP component most frequently studied in relation with the processing of contextual and lexical meaning. It is generated by several types of visual and auditory stimuli such as written or spoken words, pseudowords, drawings, faces, sounds or actions (Kutas and Hillyard 1980; Kutas and Federmeier 2011). Specifically, meaningless stimuli, like pseudowords, have been found to produce more negative amplitudes than meaningful stimuli, such as words, on the N400 component. This differential effect of the two types of stimuli has been observed in different tasks such as semantic categorization (Ziegler et al. 1997), semantic decision (Bentin et al. 1999), and lexical decision tasks (Carreiras et al. 2005; Fonseca et al. 2006; Wang and Yuan 2008). It can thus be concluded that the N400 component reflects differences in semantic processing between words and pseudowords.

Providing meaning to pseudowords by associating them with pictures and definitions or by embedding them in meaningful sentences modulates N400 amplitude (Dobel et al. 2010; Perfetti et al. 2005; Borovsky et al. 2010; Frishkoff et al. 2010). For instance, Mestres-Missé et al. (2007) have shown that readers were able to assign meaning to pseudowords when they repeatedly appeared in appropriate and restrictive sentence contexts. Indeed, at the end of the repetitions, "meaningful pseudowords" and words differed from "meaningless" pseudowords on the N400 component (see also Batterink and Neville 2011). Similar N400 effects were also found in second language acquisition: words and pseudowords from a second-language differed on N400 amplitude in a lexical decision task, indicating that learners discriminated the lexical status of both stimuli after semantic training (McLaughlin et al. 2004); also topographic differences on N400 were found for second-language nouns after learning as an index of second language proficiency increased (Stein et al., 2006). What these studies show is that the changes in activity observed on the N400 component after semantic training could be used as a sign of the construction of semantic features for these new stimuli.

Regarding familiarity, the effect of repeated exposure to unfamiliar stimuli has also been investigated in ERP studies using repetition and old/new paradigms. In this latter paradigm, participants are asked to report whether a word has been seen in a previous learning phase. This manipulation elicits a particular type of N400, an FN400 (Frontal N400), which starts around $300 \mathrm{~ms}$ but is distributed frontally (FN), unlike the typical centro-posterior N400. Because old/new paradigms and other recognition tests produce modulation of FN400, there is support for seeing this component as not exclusively related to semantic processes-it could also be a product of visual similarity between study and test items, and function as a marker of familiarity processes. However, the exact role of the FN400 in recognition memory remains unclear (GrohBordin et al. 2006; Rugg and Curran 2007; Van Strien et al. 2005; Voss and Federmeier 2011). In particular, there is no agreement about whether the FN400 component is exclusively related to the semantic processes involved in association or contextual tasks, or if it is also modulated by the increased familiarity of repeated stimuli, and as such should be seen as a separate component, different from N400. If the latter is true, changes in FN400 should be observed after repetition of new stimuli, confirming the relation between this component and changes to familiarity processes following repetition.

Furthermore, FN400 has been dissociated from the Late Positive Complex (LPC), another ERP component with a central-posterior distribution traditionally related to episodic memory processes and recollection of previously presented information (Rugg and Curran 2007; Rugg and Yonelinas 2003). It has been stated that both familiarity and recollection processes contribute to recognition memory performance. In particular, using repetition and old/ new paradigms, the amplitude of this late component (500-700 ms after stimulus onset) with a central-posterior distribution has been found to be associated with encoding and strengthening of memory traces, which allow for the retrieval and recognition of previously encoded information (Van Strien et al. 2005; Batterink and Neville 2011; Mitchell et al. 1993; Van Petten et al. 1991).

The present study aims to determine whether increased contextual familiarity of pseudowords leads to a matching cerebral activity for words and pseudowords as indicated by a decrease in the lexicality effect. The increase in familiarity was achieved by repeating exposure to pseudowords in a lexical decision task. In particular, we aimed to test whether the decrease in the lexicality effect 
occurs on FN400 or LPC components. It was expected that repeated experience with pseudowords and the resulting increase in familiarity with those items would allow the reader to pass from a sublexical reading procedure, based on activation of letters, syllables or other structures, to a strategy in which these units would be directly connected to a word-like trace. On the one hand, it was expected that FN400 would not change after repetition of meaningless pseudowords, with the lexicality effect persisting in this component despite repetition, thereby ruling the FN4000 component out as an index of familiarity processes. If, on the contrary, there was a link between FN400 and familiarity processes, the lexicality effect should decrease in this component due to the improved familiarity of stimuli following repetition. On the other hand, it was expected that the LPC component would show an increase of amplitude across repetitions as other studies have reported. The consequent decrease in the lexicality effect would indicate that repetition leads to the reinforcement of the memory trace associated to pseudowords and thereby to the improvement of the retrieval and recognition of these meaningless stimuli. To address these hypotheses on the modulation of the lexicality effect, we adopted the "classical" ERP approach of studying amplitude differences between conditions, using a cluster-based random permutation method (Maris and Oostenveld 2007). With this approach, we were able to determine which ERP components (FN400 and/or LPC) were sensitive to the lexicality effect (difference in amplitude between words and pseudowords) and to variations in this effect as a result of the repeated exposure to the same pseudowords.

A second aim of the present study was to estimate the topography (and underlying neural sources) that best covaried with the increase in pseudowords familiarity. To that end, we combined Topographical analysis of the covariance (TANCOVA) and Local Auto-Regressive Average (LAURA) source estimation methods (Koenig et al. 2008). Unlike the above classical approach, the TANCOVA method allowed us to deduct which specific scalp field configurations (topography) were varying linearly in response to repeated exposition to the same pseudoword stimuli. Furthermore, covariance maps provided by this method could be interpreted as representing the scalp field produced by the intracerebral sources that account for the effects of repetition, thus allowing us to can submit these maps directly to source localization methods to estimate the specific set of likely neural generators that account for these effects. Our specific predictions for this approach were the following. First, FN400 topography and its neural sources were not expected to be modulated by increasing familiarity of repeated meaningless pseudowords, which would allow us to discard the claim that this component is related to familiarity processes. Second, we did expect that
LPC topography and its neural sources would be affected by repetition of these items, in keeping with the hypothesis that there is a link between this component and memory and recollection processes.

\section{Materials and Methods}

\section{Participants}

Twenty-three undergraduate Psychology students (6 males) at La Laguna University took part in the experiment for course credit. All of them were right-handed native speakers of Spanish with an average score of 0.76 in the reduced version of the Edinburgh Handless Inventory (Oldfield 1971). Their mean age was 21.04 years. All participants had normal or corrected-to-normal vision and reported no neurological or psychiatric disorders.

\section{Stimuli}

Two hundred and twenty-four stimuli were presented to each participant in six different blocks. Sixty-four of them were experimental words consisting of familiar words taken from the Spanish lexicon and presented in two experimental blocks - half of them were presented in the first block and the other half in the last one, and therefore, no words were repeated. One hundred twenty-eight stimuli were filler words consisting of familiar words taken from the Spanish lexicon which formed part of four control blocks, from the second to the fifth. Finally, thirty-two stimuli were pseudowords observing the phonotactic and orthotactic rules of Spanish, and these were repeated, forming part of all six blocks of the task. Experimental words and pseudowords were matched in length (i.e., number of letters and syllables), number of orthographic neighbors, lexical frequency (LEXESP, Gallés 2000), bigram frequency (token type) and frequency (token type) of first syllable (BuscaPalabras, Davis and Perea 2005), as shown in Table 1. This matching avoided the influence of sublexical variables on other early electrophysiological components, such as the N1/P1 complex or P200 which are sensitive to attentional and orthographic processing of visual stimuli (Allison et al. 1994; Bentin et al. 1999; Hillyard et al. 1998; Nobre et al. 1994).

\section{Procedure}

Participants were seated in a silent room in the Neurocog laboratory at the University of La Laguna. After receiving verbal information, participants read the task instructions on the screen. They were allowed to take a break after each block. To prevent motor artifacts, participants were advised 
Table 1 Matching means of each variable through the experimental conditions (Words in the first and sixth block and Pseudowords) and means of each variable controlled through the filler blocks (Words in the second, third, fourth and fifth block)

\begin{tabular}{lllllrr}
\hline & $\begin{array}{l}\text { Length } \\
\text { (letters) }\end{array}$ & $\begin{array}{l}\text { Length } \\
\text { (syllables) }\end{array}$ & $\begin{array}{l}\text { Orthographic } \\
\text { neighbors }\end{array}$ & $\begin{array}{l}\text { Lexical } \\
\text { frequency }\end{array}$ & $\begin{array}{l}\text { Bigram } \\
\text { frequency }\end{array}$ & $\begin{array}{r}\text { 1st syllable } \\
\text { frequency }\end{array}$ \\
\hline Words 1st Block & 5.38 & 2.25 & 2.03 & 67.44 & 511.03 & 296.89 \\
Words 6th Block & 5.47 & 2.25 & 2.03 & 63.28 & 523.48 & 296.84 \\
Pseudowords & 5.34 & 2.26 & 2.16 & & 509.01 & 291.35 \\
Words 2nd Block & 5.03 & 2.37 & 5.3 & 67.67 & 747.32 & $2,059.79$ \\
Words 3rd Block & 5.2 & 2.1 & 4.73 & 53.86 & $1,004.46$ & 933.11 \\
Words 4th Block & 5.07 & 2.17 & 2.53 & 54.51 & 743.96 & 668.33 \\
Words 5th Block & 5.31 & 2.34 & 4.09 & 60.26 & 868.26 & $2,225.31$ \\
\hline
\end{tabular}

to avoid eye blinks only after the fixation point at each trial. Once the instructions were understood by participants, five words and five pseudowords were presented as training trials in a randomized order. Immediately afterwards and without transition, the experimental trials started. Participants had to decide if the stimulus presented was a word (pressing with their right hand a key labeled "SI") or not (pressing with their left hand a key labeled "NO"). Stimuli were displayed in black Verdana 18 point letters at the center of the screen, over white background, with the experimental software E-prime 2.0 (Schneider et al. 2002).

Time responses and accuracy for each participant were registered during this lexical decision task. Each block consisted of a total of sixty-four trials and lasted approximately $5 \mathrm{~min}$. The sequence of events in all trials was as follows. Each trial began with a fixation point presented for $1000 \mathrm{~ms}$. Then, a stimulus (experimental word, filler word or pseudoword) appeared and remained on the screen until the participant responded. At that moment, a blank screen was displayed for $500 \mathrm{~ms}$ followed by the instruction "parpadea" (blink now) lasting $1000 \mathrm{~ms}$ and another blank screen for $500 \mathrm{~ms}$. The duration of each trial depended on the time the participant took to respond, but lasted approximately $4 \mathrm{~s}$. All stimuli were presented randomized within each block.

\section{EEG Recording and Preprocessing}

EEG and EOG signals were recorded using $64 \mathrm{Ag} / \mathrm{AgCl}$ electrodes mounted in elastic Quick-caps (Neuromedial Supplies, Compumedics, Inc., Charlotte) according to the system 10/20 (Jasper 1958). A cephalic reference was taken (all electrodes were referenced to vertex), and two other electrodes were placed on mastoid bones. The EEG signal was re-referenced off-line to the mean activity in these two mastoid electrodes in keeping with the montage most typically used to evaluate N400 and LPC components. In order to monitor ocular movements and blinks, additional electrodes were placed on the external canthus of both eyes and on the left infra-orbital and supraorbital cantus. The inter-electrodes impedance was kept below
$10 \mathrm{k} \Omega$. EEG and EOG signals were amplified and digitized at a $500 \mathrm{~Hz}$ sampling rate using a SynAmps ${ }^{2}$ amplifier (Neuromedial Supplies, Compumedics, Inc., Charlotte). High and low pass filters were set at 0.05 and $100 \mathrm{~Hz}$, respectively. An additional $50 \mathrm{~Hz}$ Notch filter was applied.

EEG data pre-processing was conducted using Edit 4.5 (Neuroscan, Compumedics Inc., Charlotte). EEG data epochs between -200 to $800 \mathrm{~ms}$ poststimulus onset were extracted and submitted to the following artifact rejection procedure. First, epochs showing amplitude values exceeding $\pm 70 \mu \mathrm{V}$ in vertical and horizontal EOG channels were automatically removed. Further, a manual cleaning was carried out to ensure the complete removal of all artifacts. ERPs were then computed by averaging remaining epochs per subject and condition. The number of epochs used to compute ERPs was practically the same for all experimental conditions (from the first to the sixth experimental block, pseudowords: 26, 28, 30, 25, 29, and 26; words: 29, 24, 31, 27, 27, and 28). Baseline correction was carried out using the 200 -ms period preceding stimulus onset.

\section{Statistical Analyses}

\section{Behavioral Analysis}

For behavioral data, mean reaction times (RTs) and errors were analyzed with repeated-measures ANOVAs with Lexicality (experimental words vs. pseudowords) and Block (first vs. sixth) as within-subject factors. Additionally, for pseudowords, a regression coefficient $\left(R^{2}\right)$ was computed to estimate the amount of variance of each behavioral measure (RTs and errors) that was explained by repetitions. This regression analysis explores, beyond the ANOVAs, the causal relation between pseudoword repetition throughout the blocks and RTs and errors, clarifying how the speed and accuracy achieved by participants are modified as result of repetitions. Trials in each block with incorrect responses, or with response latencies over 2 standard deviations were excluded from all these analyses. In addition, one item from the first experimental block was 
eliminated because its incorrect presentation caused participants to err.

\section{ERP Analysis}

Lexicality effects on ERPs were studied by analyzing the differences between words and pseudowords occurring in the first and the last experimental block. The resulting $2 \times 2$ design, with lexicality (word, pseudoword) and experimental block (first, sixth block) as within-subject factors, was evaluated using the cluster-based random permutation method implemented in Fieldtrip (Maris and Oostenveld 2007; Oostenveld et al. 2011). This method deals with the multiple comparisons in space and time by identifying, over the whole ERP segment (here, 400 time points $\times 62$ channels $=24,800$ sample points), clusters of significant differences between conditions (sample points in close spatial and temporal proximity), while effectively controlling for type 1 error. Here is a brief description.

In a first step, cluster-level statistics are computed as follows. For every sample (time by electrode) point, the difference between conditions is quantified by means of a dependent sample $t$ test. From this comparison, all sample points below or equal to a predetermined threshold (e.g., $\alpha$ level of 0.05 ) are selected, and clusters formed on the basis of spatial and temporal adjacency. For each cluster with a minimum of spatially adjacent sample points (here, 3), a single measure of the observed cluster effect size (the cluster-level statistic) is finally calculated by taking the sum of all the individual $t$ values within it. Next, in a second step, a null distribution of cluster-level statistics is computed using a random partition procedure. With this procedure, subject ERPs segments are randomly assigned to experimental conditions a number of times (here, 2,000). After each randomization, cluster-level statistics are then calculated as above, and the one with the largest effect size (sum of $t$ values) enters into the null distribution. The proportion of cases in which the values of this distribution are larger than the observed cluster-level statistic represents the probability of the null hypothesis, which is computed for each observed cluster. If this probability is below or equal to a predetermined threshold (here, $5 \%$ ) then the null hypothesis is rejected, and the observed cluster considered significant.

This statistical method allows only for pair-wise comparisons. Therefore, certain prior calculations were performed in order to evaluate the main effects and the interaction of the $2 \times 2$ design. Regarding the main effects, an average of the two conditions corresponding to the same level of each of the two factors was calculated for each subject, and comparisons performed using these two averages. For example, for the main effect of lexicality, the average between the words in the first block and the words in the sixth block formed the level 'word' of the factor, and was compared to the one calculated for the level 'pseudoword'. For testing the interaction, word minus pseudoword difference waveforms were computed for each block separately. Then, these difference waveforms were statistically compared to each other. Additional comparisons were also conducted to decompose the outcome (significant clusters) of the two main effects and the interaction. In these comparisons, mean amplitudes over the time windows of the significant cluster/s served as input to the test.

\section{Topographical Analysis of Covariance}

TANCOVA, introduced by Koenig et al. (2008), was used to identify the significant time points in which global scalp field potentials for pseudoword covaried with the external variable repetition (six levels). This analysis determines whether such covariance between ERP topography and an external variable is above chance levels. Unlike "classical" ERP analyses based on amplitude differences, this approach was expected to provide information on which scalp field configurations (topographies) were linearly varying their strength across repetition. Next is a brief description of this statistical method.

In a first step, the covariance between the potentials at each electrode and the external variable is computed, and an appropriate measure of effect size for the resulting covariance map calculated. Since the higher the association between source activity and external variable, the higher the global strength of the covariance map, the global field power (GFP, Lehmann and Skrandies 1980) of the covariance map serves as effect size. GFP represents a single, reference-independent measure of the strength of the scalp potentials field, which is statistically equivalent to the standard deviation of the potentials at all electrodes (e.g. Murray et al. 2008). In a second step, a null distribution of covariance maps and their corresponding GFP is obtained by randomly assigning (here, 5,000 times) ERP segments to the external variable. The percentage of cases in which the GFP obtained after randomization is larger than the observed GFP represents the probability of the null hypothesis. This null hypothesis is finally evaluated, so that if its probability is equal or below a determined threshold then it is rejected, and consequently the alternative hypothesis accepted - that is, the observed effect size (GFP of the covariance map) is considered significant.

This method is applied for each time point of the ERP segments separately (400 time points), and hence it does not directly address the multiple comparisons. To effectively control for the type 1 error related to these multiple comparisons, the threshold used to evaluate the probability 
of the null hypothesis was estimated based on the false discovery rate (FDR), with a FDR criterion of $5 \%$ (Genovese et al. 2002). The FDR threshold was obtained by comparing the distribution of observed $p$ values with the distribution of accepted "false positives" (incorrect rejections of the null hypothesis), which was estimated by multiplying the expected distribution of the $p$ values under the null hypothesis by the FDR criterion. Once the two distributions were ordered from the smallest to the largest value, the first observed $p$ value below or equal to the corresponding value of the distribution of accepted "false positives" was taken to represent the FDR threshold such that only observed $p$ values below this threshold were considered to represent "true discoveries" (correct rejections of the null hypothesis).

\section{Source Localization}

The covariance maps obtained with TANCOVA analysis represent a linear transformation of the original ERP topography, and hence can be directly submitted to source localization methods (Koenig et al. 2008; Pedroni et al. 2011). Brain sources of the covariance maps were estimated using the distributed source estimation method LAURA (Grave de Peralta Menendez et al. 2001), implemented in Cartool software (Brunet et al. 2011). Distributed localization algorithms are generally preferred to more classical dipole modelling when there is no clear prediction about the location and the number of involved neural sources, as it is the case in the present study (e.g. Michel et al. 2004). The choice for LAURA source estimation was motivated by its extensive and successful use in prior research and its localization precision, which has been evaluated in modelling (de Peralta Menendez and Andino 1999) and epileptic patient research (e.g. Groening et al. 2009).

The solution space was calculated on a realistic head model that included 4,025 nodes, defined in regular distances within the gray matter of a standard MRI (Montreal Neurological Institute's average brain). Current density magnitudes (ampere per square millimeter) at each node were calculated for the time windows showing significant results in the TANCOVA analysis. The resulting magnitudes represent the intracerebral generators of the scalp field data accounting for the effects of the variable repetition in each time window.

\section{Results}

Behavioral Results

Mean reaction times and errors are presented in Table 2. ANOVAs across participants F1 and items F2 were carried
Table 2 Mean RTs for Words and Pseudowords presented in the first and in the sixth block (analysis by participants)

\begin{tabular}{lll}
\hline & \multicolumn{2}{l}{ Mean RTs and errors } \\
\cline { 2 - 3 } & 1st & 6th \\
\hline Words & $680.38(0.26)$ & $654.22(0.47)$ \\
Pseudowords & $976.69(1.26)$ & $720.03(0.52)$ \\
\hline
\end{tabular}

Mean of errors for the same stimuli and conditions is showed in brackets

out on latencies. Regarding item based ANOVAs, the analyses were run across the averages of all individual test items in order to control for the items' variability so that results could be generalized to the entire set of items as well as participants (Clark 1973). The analysis revealed a main significant effect of Lexicality, showing that mean RTs for pseudowords were longer than for words. A main effect of Block was also obtained, because latencies for first presentation of stimuli were longer than for the sixth. In addition, the interaction between Lexicality and Block was statistically significant. Follow up pair-wise comparisons tested by one way ANOVAs revealed that the $26.17 \mathrm{~ms}$ difference between words presented in the first and sixth block was not statistically significant, in contrast with the $256.66 \mathrm{~ms}$ difference between first and sixth block for pseudowords which was significant. Additionally the difference between RTs for words and pseudowords was significant both in the first block (680.38 and $976.68 \mathrm{~ms}$ respectively) and in the last block after five repetitions of pseudowords (654.21 and $720.02 \mathrm{~ms}$, respectively). Although in the sixth block there remains a difference between words and pseudowords, the interaction of Lexicality by Block indicates a significant reduction on RTs for pseudowords (see Table 3 for detailed statistical result).

The ANOVAs on errors revealed a main effect of Lexicality and a significant Lexicality by Block interaction. No Block effect emerged. Follow up analyses revealed that participants committed more errors on pseudowords in the first block than in the sixth (1.26 vs. 0.52) although this result was only found in the analysis by participants. This effect was not found for words in the first and sixth block (0.26 vs. 0.47). Additional comparisons revealed significant differences between errors on words and pseudowords displayed in the first block ( 0.26 vs. 1.26$)$ but not in the sixth block (0.47 vs. 0.52). Therefore, participants are equally competent for both types of stimuli in the last block (see Table 3).

The regression analysis conducted on pseudowords' mean RTs of each participant across the six blocks of the task revealed a significant linear trend $\left[R^{2}=0.118\right.$, $F(1,136)=19.28, p<0.001]$. Similarly, a significant linear trend was obtained for errors made by participants on pseudowords across the six blocks $\left[R^{2}=0.057\right.$, $F(1,136)=9.31, p<0.01]$. The improvement in speed 
Table 3 Statistical results (both participants and items analyses) of the behavioral data: general ANOVAs (Lexicality and Block factors, and Lexicality by Block interaction) and follow up comparisons (Words in the first vs. sixth block, Pseudowords in the first vs. sixth block and Words vs. Pseudowords in the first and in the sixth block)

\begin{tabular}{|c|c|c|c|c|c|c|c|c|}
\hline & \multicolumn{4}{|c|}{ Participants } & \multicolumn{4}{|l|}{ Items } \\
\hline & $\mathrm{F} 1(1,22)$ & $p$ & $\eta^{2}$ & $1-\beta$ & $\mathrm{F} 2(1,124)$ & $p$ & $\eta^{2}$ & $1-\beta$ \\
\hline \multicolumn{9}{|l|}{ Mean RTs $(2 \times 2$ ANOVA $)$} \\
\hline Lexicality & 17.45 & $<0.001$ & 0.44 & 0.97 & 280.65 & $<0.001$ & 0.69 & 1 \\
\hline Block & 10.88 & $<0.01$ & 0.33 & 0.88 & 170.49 & $<0.001$ & 0.57 & 1 \\
\hline Interaction & 11.39 & $<0.01$ & 0.34 & 0.89 & 118.43 & $<0.001$ & 0.48 & 1 \\
\hline Follow up comparisons & & & & & $\mathrm{F} 2(1,62)$ & & & \\
\hline Words 1st vs. 6th block & 2.17 & $>0.05$ & 0.09 & 0.29 & 3.77 & $>0.05$ & 0.05 & 0.48 \\
\hline Pseudowords 1st vs. 6th block & 11.57 & $<0.01$ & 0.34 & 0.90 & 208.50 & $<0.001$ & 0.77 & 1 \\
\hline Words vs. Pseudowords 1st block & 15.04 & $<0.01$ & 0.40 & 0.95 & 282.23 & $<0.001$ & 0.82 & 1 \\
\hline Words vs. Pseudowords 6th block & 17.14 & $<0.001$ & 0.43 & 0.97 & 26.62 & $<0.001$ & 0.30 & 0.99 \\
\hline Mean of Errors $(2 \times 2$ ANOVA $)$ & & & & & $\mathrm{F} 2(1,124)$ & & & \\
\hline Lexicality & 5.56 & $<0.05$ & 0.20 & 0.61 & 5.47 & $<0.05$ & 0.04 & 0.64 \\
\hline Block & 2.09 & $>0.05$ & 0.08 & 0.28 & 0.95 & $>0.05$ & 0.00 & 0.16 \\
\hline Interaction & 17.17 & $<0.001$ & 0.43 & 0.97 & 5.47 & $<0.05$ & 0.04 & 0.64 \\
\hline Follow up comparisons & & & & & $\mathrm{F} 2(1,62)$ & & & \\
\hline Words 1 st vs. 6th block & 1.50 & $>0.05$ & 0.06 & 0.21 & 1.71 & $>0.05$ & 0.02 & 0.25 \\
\hline Pseudowords 1st vs. 6th block & 9.08 & $<0.01$ & 0.29 & 0.82 & 3.77 & $>0.05$ & 0.05 & 0.48 \\
\hline Words vs. Pseudowords 1st block & 14.05 & $<0.01$ & 0.39 & 0.94 & 9.46 & $<0.01$ & 0.13 & 0.85 \\
\hline Words vs. Pseudowords 6th block & 0.03 & $>0.05$ & 0.002 & 0.05 & 0 & $>0.05$ & 0.00 & 0.054 \\
\hline
\end{tabular}
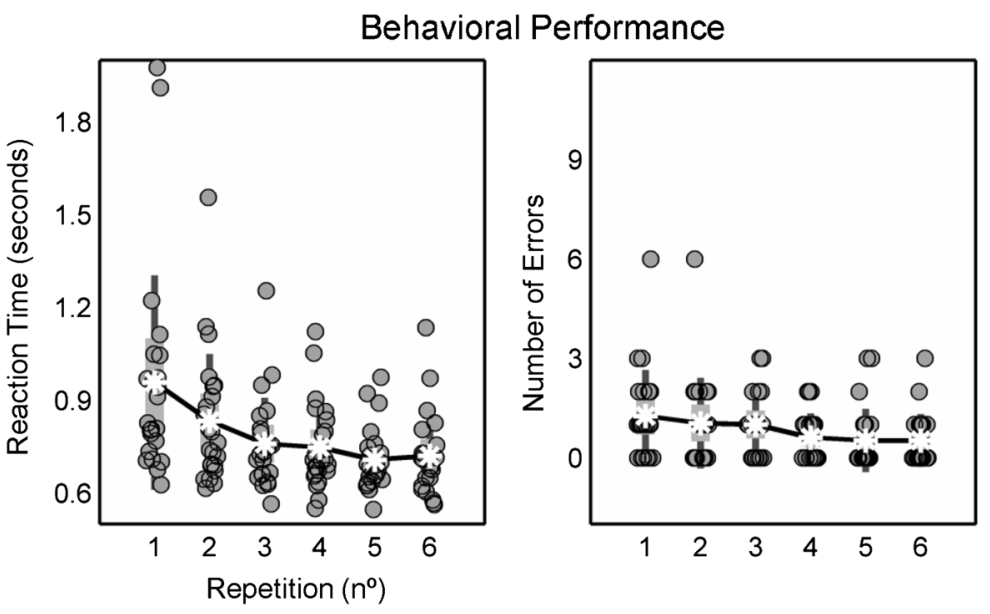

Late Positive Component

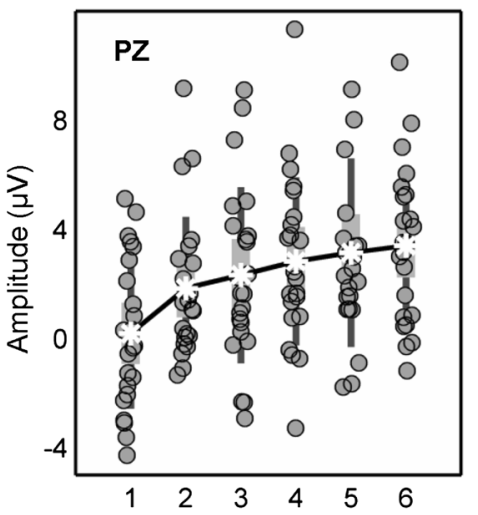

Fig. 1 Mean values for reaction times (RTs), number of errors, and amplitudes at a representative electrode (PZ) of the late positive component amplitude (LPC, between 468 and 634 ms). Grey shaded dots represent mean values for each participant. White asterisks

and accuracy for pseudowords is thus explained by the variable Repetitions (See Fig. 1).

\section{ERP Results}

Figure 2 displays waveforms (panel a) and results of the ERP analyses with the cluster-based random permutation represent total mean values. As shown by dark lines, both RTs and number of errors tend to reduce across repetitions, while LPC amplitudes tend to increase with repetitions

procedure (panel b). Tests on the main effect of lexicality resulted in one significant cluster $(p<0.001$, Fig. $2 b)$. This cluster extended approximately between 302 and $520 \mathrm{~ms}$, showing a fronto-central distribution, and revealed less positive amplitude for pseudowords than for words. Follow up comparisons (using mean amplitude during the cluster time window, 302-520 ms) confirmed the difference 
A
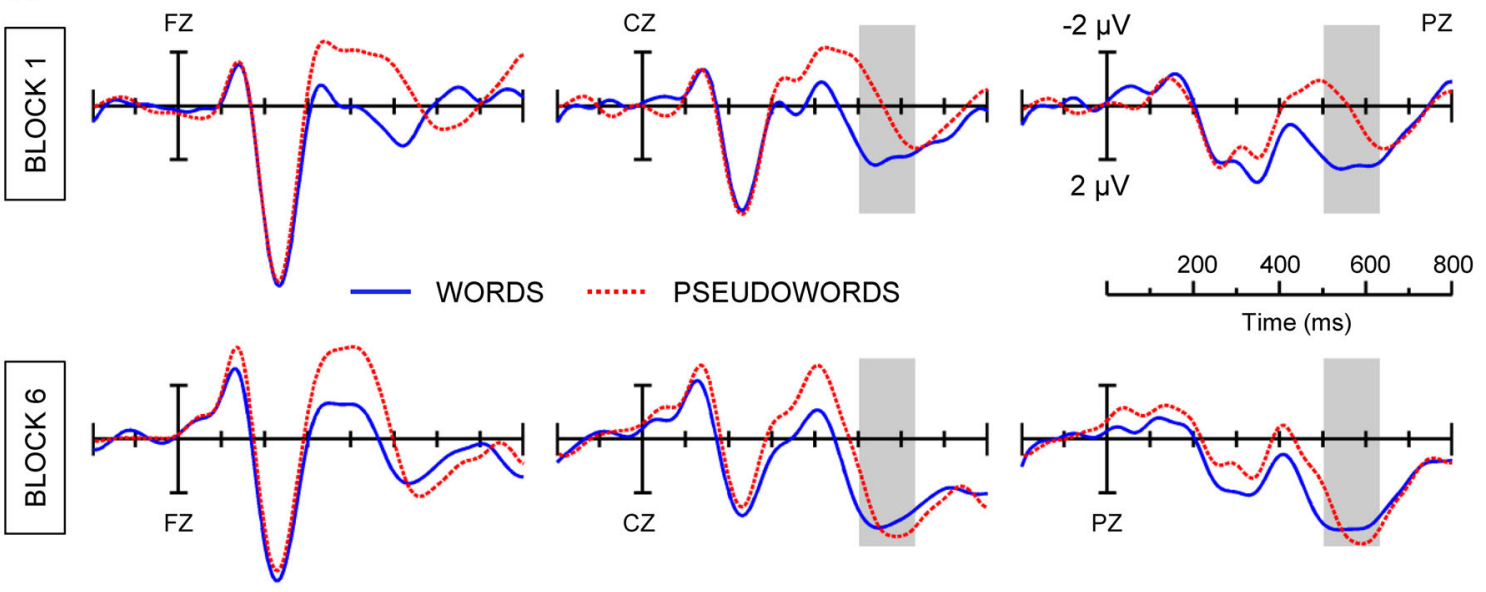

B
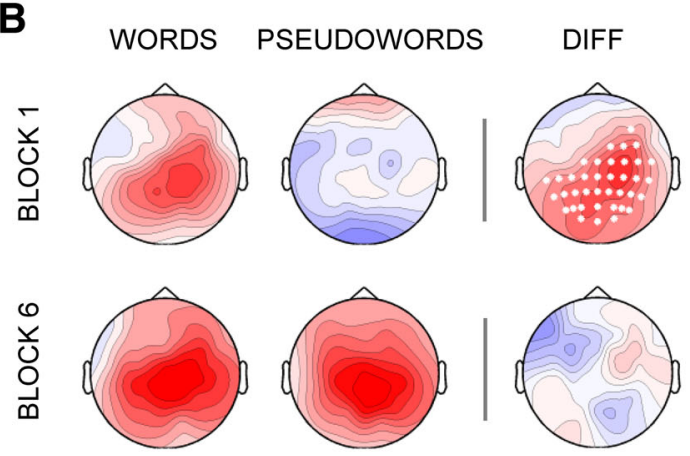

DIFF

\section{WORDS PSEUDOWORDS}
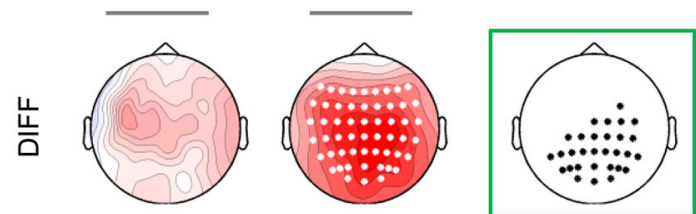

Interaction Effect : LPC (500-618 ms)

Fig. 2 Upper half a Averaged ERP waveforms at medial scalp sites (FZ, CZ, PZ electrodes) for words and pseudowords in the first and sixth experimental block. Grey shaded areas highlight the time window of the significant interaction between stimuli and block type. Follow-up comparisons confirmed differences during this time window (500-618 ms) between words and pseudowords in the first block but not in the sixth block, due to pseudoword amplitude changes across blocks. Comparisons showed amplitude differences between first and sixth block for pseudowords but not for words;

between words and pseudowords occurring within both the first $(p<0.005)$ and the last experimental block $(p<0.005)$. Latency and topographic characteristic of the cluster suggest this cluster is probably reflecting a modulation of the N400-like component.

Testing of the main effect of block also yielded one significant cluster $(p<0.001)$. In this case, the cluster extended

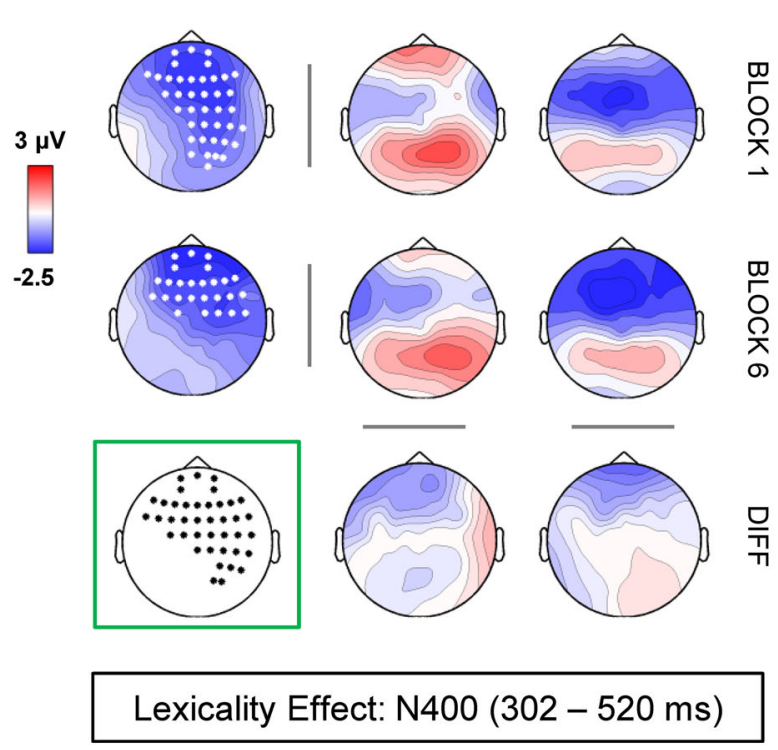

pseudowords were more positive in the last block than in the first. Lower half $\mathbf{b}$ overview of cluster analysis results. Maps framed in green represent the sites belonging to the significant clusters for the interaction and for the lexicality effect. Maps under (or to the left/ right of) the label DIFF depict scalp distribution of the differences between conditions. White points highlight the scalp sites corresponding to the significant cluster for these differences. All other maps represent the topography of the ERP activity for each condition (Color figure online)

between 468 and $634 \mathrm{~ms}$, showing a central-posterior distribution, and revealed less positive amplitude for the first block stimuli than the last block stimuli. However, further comparisons showed that amplitude differences between blocks reached significance only for pseudowords $(p<0.001)$. Latency and topographic characteristics suggest that this effect is likely to affect the LPC component. 
Testing of the interaction produced a significant cluster ( $p<0.005$, Fig. 2b) with latency (between 500 and $618 \mathrm{~ms}$ ) and topographic characteristics (central and posterior sites) similar to those for the main effect of block. This similarity suggests the interaction is probably also reflecting a modulation of the LPC component, and thereby qualifying the main effect of block. Follow up comparisons revealed the effects in this cluster were due to the less positive amplitude of pseudowords in the first block than of the other conditions (see Fig. 1). Such reduced LPC amplitude was responsible for the appearance of both an effect of lexicality in the first block $(p<0.001)$ and an effect of block for pseudowords $(p<0.001)$.

In sum, two spatiotemporal loci of effects emerged after the statistical analyses of ERPs. In the first one, which appeared in a cluster between 302 and $520 \mathrm{~ms}$ (reflecting the unfolding of a N400-like component), similar differences between words and pseudowords were found for both the first (first presentation of pseudowords) and the last experimental block (sixth repetition of pseudowords). In the second one, which appeared in a cluster between 468 and $634 \mathrm{~ms}$ (LPC component), pseudowords showed a shift in amplitude (more positive) from the first to the sixth repetition, which contributed to the absence of the lexicality effect in the last experimental block.

\section{ERP-Repetition Covariance Maps: Topography and Neural Sources}

The above ERP analyses suggest that the repetition of pseudowords likely modulated the activity of the LPC component. Nevertheless, to better evaluate the impact of repetition on the electrophysiological brain response to pseudowords, we conducted a topographical analysis of the covariance between the repetition of pseudowords and the ERP activity (Fig. 3a, where $p$ values represent, inversely, the significance level of the covariance map), and estimated the likely sources of the time periods with significant topographic covariance (Fig. 3b). The TANCOVA analysis revealed significantly covarying (below FDR threshold, $p<0.004$, Fig. 3a) ERP topographies in the time windows of $110-142 \mathrm{~ms}$ and $570-630 \mathrm{~ms}$, indicating that, as the number of repetitions increased (from the first to the sixth block), so did the strength (global field power, GFP) of the scalp field configuration (topography) observed in these two periods. The topography of the early window revealed that the repetition variable covaried with negative ERP activity at anterior sites and with positive ERP activity at right posterior sites. This topography, along with the time period of the effect, suggests the covariance reflects changes in the activity of the N1/P1 ERP complex. Points of maximal current source magnitude for the covariance map in this time window (Fig. 3b) were found in the right lingual gyrus (GL, maximum at $\mathrm{x}=17, \mathrm{y}=-65, \mathrm{y}=$ $-4 \mathrm{~mm}$ using the coordinate system of Talairach and Tournoux, 1988; corresponding to BA 19) and the right middle temporal gyrus (MTG, maximum at $\mathrm{x}=58, \mathrm{y}=3$, $\mathrm{y}=-9 \mathrm{~mm}$; BA 21). Positive central and posterior ERP activity characterized the later window with significant covariance. In accordance with the ERP analysis, this covarying topography revealed changes in the activity of the LPC component due to pseudoword repetition across blocks. Points of maximal current source magnitude were found for this time window (Fig. 3b) in the left anterior superior temporal gyrus (STG, maximum at $\mathrm{x}=-31$, $\mathrm{y}=22, \mathrm{z}=-38 \mathrm{~mm}$; BA 38), the left inferior frontal gyrus (LIFG, maximum at $\mathrm{x}=-31, \mathrm{y}=29, \mathrm{z}=-21$; BA 47), and the right superior frontal gyrus (SFG, maximum at $\mathrm{x}=30, \mathrm{y}=70, \mathrm{z}=-12$; $\mathrm{BA} 10)$.

The TANCOVA analysis was also conducted for words in order to discard the possibility that covarying ERP maps of pseudowords were related to a general change in activity, and consequently not directly associated to repetitions. Unlike pseudowords, words were different for each experimental block and hence, should not show changes in brain activity due to stimulus repetition. As the distribution of $p$ values from Fig. 3a shows, there was no significant period in which words' ERP activity covaried with the variable block. Thus, TANCOVA results for pseudowords can be specifically and reliably attributed to the influence of repetition (see "Discussion").

\section{Discussion}

The present study aimed to test whether pseudoword repetition leads to a decrease in the lexicality effect, and whether this is accompanied by a modulation in the activity of the FN400 and the LPC. Behavioral data showed that, for pseudowords, reaction times and errors were reduced by repetition, but this manipulation did not completely eliminate the lexicality effect. Thus, the repetition of meaningless pseudowords improved their processing so that they reached a processing level equivalent to that of words in terms of response accuracy but not entirely in terms of speed. At the beginning of the lexical decision task, pseudoword discrimination was difficult and more errors were committed because words and pseudowords were matched on their sublexical composition (bigrams and syllables frequency) which prevented pseudoword facilitation through bottom-up processes; however, memory trace formed across repetitions seems to have improved the discrimination and lexical categorization of pseudowords up to a level of accuracy similar to that of words. With regards to speed in the processing of pseudowords, the lexicality effect was maintained across the task because 


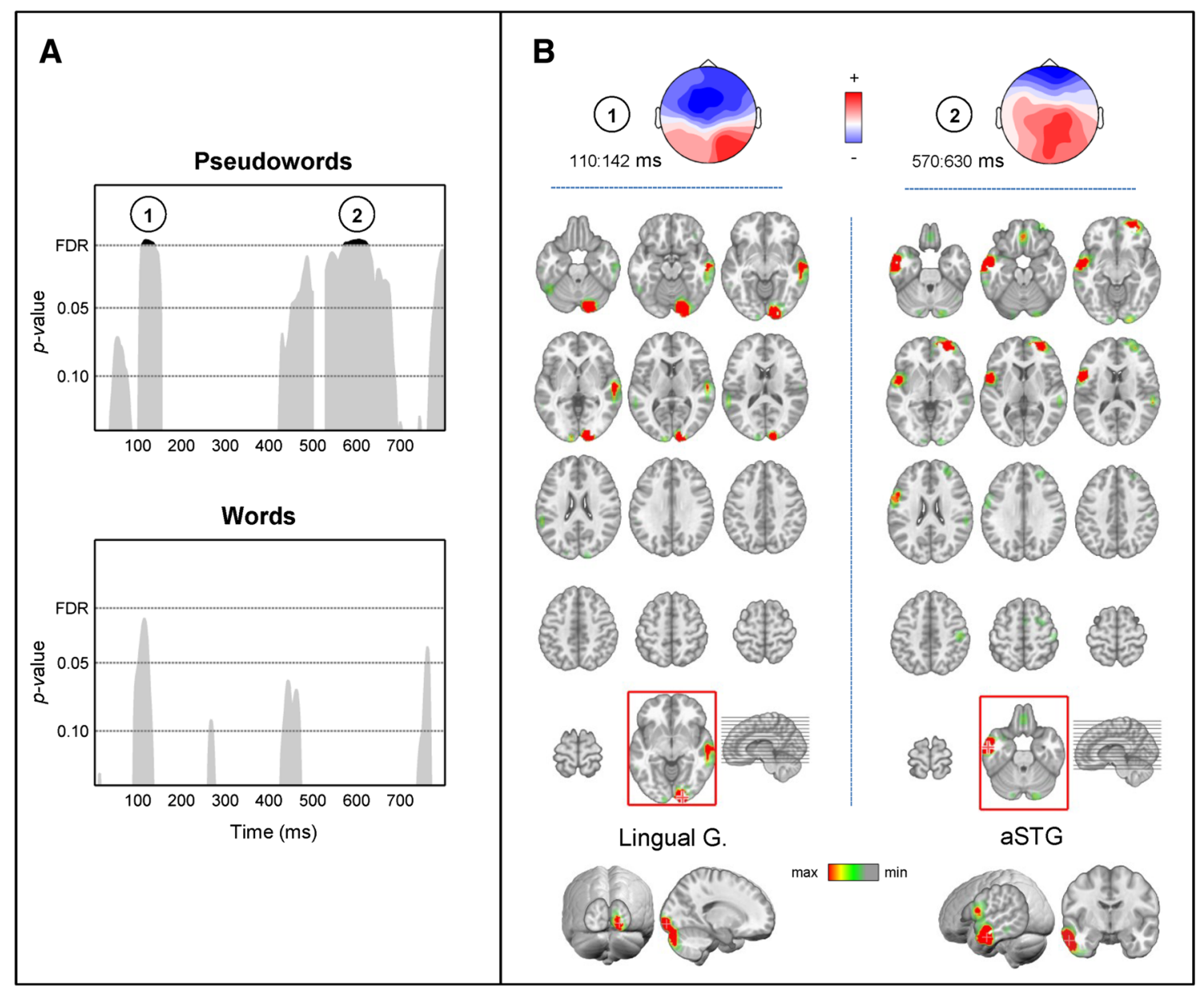

Fig. 3 TANCOVA results overview. On the left (a), time-point by time-point significance levels of the TANCOVA analyses for pseudowords and words. The height of the area represents, inversely, the $p$ values (probability) of the null hypothesis. Black shaded areas indicate the periods in which the covariance between ERP topography and the variable repetition reached significance values below the estimated FDR threshold $(p<0.004)$. Only pseudowords showed periods of significant covariance, reflecting the variation in the strength (global field power) of the scalp field configuration during these periods as a function of the repeated exposure to these stimuli.

the semantic facilitation through top-down processes only affected words but not pseudowords. It is probable that semantic training in addition to visual repetition is needed to achieve a direct visual recognition that would decrease the pseudoword decision time enough to remove the lexicality effect completely.

Electrophysiological data also showed a modulation prompted by pseudoword repetition which was characterized by two different patterns. First, a negative component with a fronto-central distribution, FN400, showed a lexicality effect which remained unaffected by the repetition of pseudowords. This result confirms the hypothesis that FN400 is not affected by variations in familiarity produced by the repetition of stimuli and suggests that this
On the right $\mathbf{b}$, map topographies of the significant periods and their corresponding source estimations. The loci of maximal current source magnitudes (representing the maximal contribution to the covariance topography) are framed in red. Topography and timing of the second significant period are consistent with the typical topography of the LPC component. Sources in the left anterior superior temporal gyrus (aSTG), left inferior frontal gyrus (IGF) and right superior frontal gyrus (SFG) contribute to this LPC-like covariance topography (Color figure online)

component may only be sensitive to differences in stimuli based on their semantic features in memory as other authors have already argued (Voss and Federmeier 2011). Second, LPC showed a central and posterior distribution and a larger amplitude for pseudowords after each repetition (block effect), approaching the amplitude measured for words in the last presentation and, therefore, causing the lexicality effect to disappear. To the best of our knowledge, past studies reporting an increase in LPC amplitude after repetition do not report this similarity between words and pseudowords (Batterink and Neville 2011; Renoult et al. 2012; Van Strien et al. 2005). The remaining lexicality effect in the Batterink and Neville study was explained by the semantic demands of the task and by the contribution of 
the memory trace formed given the task requirements (learning of pseudoword meaning). In such a task with a semantic demand, the memory trace built by repetition of meaningful pseudowords contributed to the comprehension of these stimuli to a greater extent than words. The reason is that pseudowords implied more active retrieval processes and, therefore, elicited larger LPC amplitudes than words because words have already well established meanings and do not require such processes. However, in the present task, where the task is orthographic, the LPC amplitudes for repeated pseudowords increased until they reached the amplitude of words. This result shows that the LPC component may be related to the formation and strengthening of memory traces, which in the present study would have contributed to reach a level of recognition and lexical decision for the repeated pseudowords similar to that observed for words (as reflected in the improvement of speed and accuracy for these stimuli).

Although some authors report a repetition effect on FN400, relating this component more to familiarity than to semantic processes (Bridger et al. 2012; Laszlo and Federmeier 2007; Laszlo et al. 2012), our results do not support this claim. Instead, repetition only affected LPC up to the point where the lexicality effect was not evident, reflecting the process of formation and strengthening of a memory trace for repeated pseudowords which improves recognition and association of these pseudowords with a given response in a lexical decision task.

It is probable that FN400 modulations could be obtained through the semantic manipulation of pseudowords. However, adding meaning to repeated pseudowords confounds the interpretation of repetition effects on FN400 preventing us from clarifying the relation between this component and familiarity processes. In this sense, the preservation of the lexicality effect on FN400 found in this study after pseudoword repetition contrasts with other studies which reported $\mathrm{N} 400$ effects. In those experiments, semantic training is provided for pseudowords and, as a result, N400 decreases to the point where the lexicality effect ultimately disappears (Batterink and Neville 2011; Mestres-Missé et al. 2007). This inconsistency is not surprising given that, in the present study, pseudowords were meaningless stimuli not associated with semantic information (this was done in order to ensure the familiarity effects were not biased by semantic effects). Similarly to previous studies, we found that pseudowords produced deeper memory traces with each repetition. These traces probably involve sub-lexical units at the beginning of the task and representations of whole words at the end. Although readers were exposed only to visual stimuli in a lexical decision task, it is possible that these traces involve phonological rather than visual representation. Overall, our findings suggest that there is some dissociation between the two electrophysiological components, FN400 and LPC, as reflected by the lexicality effect. Whereas LPC shows similar processing for words and repeated pseudowords due to the construction and reinforcement of visual memory traces for new stimuli, FN400 reflects differences in stimulus processing which were not reduced after familiarity training, probably due to the non-resolution of semantic uncertainty over the course of the experiment.

Further to previous studies related to repetition effects of new stimuli, novel and complementary data were obtained by means of topographical and neural source analyses. A covariance map showed changes in LPC caused by the repetition of pseudowords to be compatible with probable neural sources in the left inferior frontal gyrus, left superior temporal gyrus and right superior frontal gyrus, consistent with other studies examining the neural sources of LPC modulations by repetition effects (Kim et al. 2006, 2012; Taha and Khateb 2013). Interestingly, these regions have traditionally been related to phonological and control processes and are thought to be involved in the use of the grapheme-to-phoneme recoding mechanism (G-P), responsible for reading low-familiarity stimuli like pseudowords (Herbster et al. 1997; Joubert et al. 2004; Newman and Twieg 2001; Price 1998; Rumsey et al. 1997; Levy et al. 2009; Juphard et al. 2011; Ripamonti et al. 2014). Spanish is a completely regular language in terms of its G-P rules. When a pseudoword is read for the first time, the only possible way for it to be read is by applying a phonological recoding to pronounce it. However, when the stimulus becomes familiar, its processing is facilitated and the use of this mechanism is reduced. A possible interpretation of this result is that readers passed from the use of this recoding mechanism at the beginning of the lexical decision task to the direct association throughout repetition between the memory trace developed for pseudowords and the particular response to these stimuli in the task. In fact, the anterior STG has been identified as a responsible region for associations between letters and speech sounds (Van Atteveldt et al. 2007). The mechanism underlying these associations showed less activation after repetition. This effect is probably due to top-down visual effects on mapping the pseudowords to their representations, (representations whose phonological nature is not completely discarded). As can be seen in our data, the recognition of pseudowords was automated through repetition; however, this automatization did not activate the left fusiform region, specialized on visual word recognition (Cohen and Dehaene 2004). This lack of activation suggests that repetition of meaningless pseudowords is not enough to reach automatic recognition and the activation of the Visual Word Form Area exhibited by real words, which have been encountered by readers far more often and, crucially, carry semantic content.

Another significant covariance map between repetition and pseudoword ERP activity was found at the P1/N1 ERP 
complex, with the lingual gyrus as one of the probable neural sources for this covariance. This visual region has been related to the visual processing required for reading, in particular to the whole shape processing that is activated when attention is directed to overall forms, such as word profiles (Fink et al. 1997; Mechelli et al. 2000). In this line, our results indicate a better and stronger discrimination of the visual trace developed for pseudowords through repetition.

In conclusion, our results support a functional dissociation between the two FN400 and LPC components and the respective underlying processes involved in achieving wordlike processing for new stimuli after repetition. Contrary to previous assumptions, FN400 is not sensitive to familiarity processes arising from the repetition of meaningless pseudowords. A different processing of words and pseudowords persisted on this component despite repetition; which could be explained by the influence of semantic features of both meaningful and meaningless stimuli. In contrast, pseudoword repetition did affect LPC, a component that reflects the formation of memory traces, which led to improved recognition of new stimuli over the course of the task to the point that the difference between pseudowords and words was virtually eliminated. The LPC topography varied across the repetitions, with neural generators located on areas related to a phonological recoding mechanism for reading unfamiliar stimuli. Given these results, it can be said that the visual memory trace obtained by repetition improves the processing of repeated new stimuli. However, this is not enough to establish a functional role for these stimuli similar to that of words, in the linguistic system, as was reflected by the maintenance of the lexicality effect on the FN400 component and on behavioral data. The final step to completing the neural network underlying word representations in the linguistic system, besides the improvement of the visual memory traces of the new stimuli, should be the activation of semantic features. New studies that manipulate the meaning of novel stimuli apart from their visual repetition are necessary to observe the evolution of FN400 and LPC components in relation to the construction of new semantic and visual memory traces.

Acknowledgments This research was funded by the Grants PSI2012-31913 and PSI2010-15184 from the Spanish Government and supported by a predoctoral grant from the Foundation for the promotion of Applied Scientific Research and Technology in Asturias (FICYT).

\section{References}

Allison T, McCarthy G, Nobre A, Puce A, Belger A (1994) Human extrastriate visual cortex and the perception of faces, words, numbers, and colors. Cereb Cortex 4(5):544-554. doi:10.1093/ cercor/4.5.544

Batterink L, Neville H (2011) Implicit and explicit mechanisms of word learning in a narrative context: an event-related potential study. J Cogn Neurosci 23(11):3181-3196. doi:10.1162/jocn_a_ 00013

Bentin S, Mouchetant-Rostaing Y, Giard M, Echallier J, Pernier J (1999) ERP manifestations of processing printed words at different psycholinguistic levels: time course and scalp distribution. J Cogn Neurosci 11(3):235-260. doi:10.1162/ 089892999563373

Borovsky A, Kutas M, Elman J (2010) Learning to use words: eventrelated potentials index single-shot contextual word learning. Cognition 116(2):289-296. doi:10.1016/j.cognition.2010.05.004

Breitenstein C, Zwitserlood P, de Vries MH, Feldhues C, Knecht S, Dobel C (2007) Five days versus a lifetime: intense associative vocabulary training generates lexically integrated words. Restor Neurol Neurosci 25(5):493-500

Bridger EK, Bader R, Kriukova O, Unger K, Mecklinger A (2012) The FN400 is functionally distinct from the N400. Neuroimage 63(3):1334-1342. doi:10.1016/j.neuroimage.2012.07.047

Brunet D, Murray MM, Michel CM (2011) Spatiotemporal analysis of multichannel EEG: CARTOOL. Comput Intell Neurosci 2011:2. doi: $10.1155 / 2011 / 813870$

Carreiras M, Vergara M, Barber H (2005) Early event-related potential effects of syllabic processing during visual word recognition. J Cogn Neurosci 17(11):1803-1817. doi:10.1162/ 089892905774589217

Clark HH (1973) The language-as-fixed-effect fallacy: a critique of language statistics in psychological research. J Verbal Learn Verbal Behav 12(4):335-359. doi:10.1016/S0022-5371(73) 80014-3

Clay F, Bowers JS, Davis CJ, Hanley DA (2007) Teaching adults new words: the role of practice and consolidation. J Exp Psychol Learn Mem Cogn 33(5):970. doi:10.1037/0278-7393.33.5.970

Cohen L, Dehaene S (2004) Specialization within the ventral stream: the case for the visual word form area. Neuroimage 22(1): 466-476. doi:10.1016/j.neuroimage.2003.12.049

Davis CJ, Perea M (2005) BuscaPalabras: a program for deriving orthographic and phonological neighborhood statistics and other psycholinguistic indices in Spanish. Behav Res Methods 37(4): 665-671. doi:10.3758/BF03192738

de Peralta Menendez RG, Andino SG, Lantz G, Michel CM, Landis T (2001) Noninvasive localization of electromagnetic epileptic activity. I. Method descriptions and simulations. Brain Topogr 14(2):131-137. doi:10.1023/A:1012944913650

de Peralta Menendez RG, Andino SG (1999) Distributed source models: standard solutions and new developments. Analysis of neurophysiological brain functioning. Springer, Berlin, pp 176-201. doi:10.1007/978-3-642-60007-4_10

Dobel C, Junghöfer M, Breitenstein C, Klauke B, Knecht S, Pantev C, Zwitserlood P (2010) New names for known things: on the association of novel word forms with existing semantic information. J Cogn Neurosci 22(6):1251-1261. doi:10.1162/jocn. 2009.21297

Dumay N, Gaskell MG (2007) Sleep-associated changes in the mental representation of spoken words. Psychol Sci 18(1):35-39. doi:10.1111/j.1467-9280.2007.01845.x

Fink GR, Halligan PW, Marshall JC, Frith CD, Frackowiak R, Dolan RJ (1997) Neural mechanisms involved in the processing of global and local aspects of hierarchically organized visual stimuli. Brain 120(10):1779-1791. doi:10.1093/brain/120.10.1779

Fonseca LC, Tedrus GM, Gilbert MAP (2006) Event related potentials during the visual discrimination of words and pseudowords by children. Arq Neuropsiquiatr 64(3A):553-558. doi:10.1590/S0004-282X2006000400002

Frishkoff GA, Perfetti CA, Collins-Thompson K (2010) Lexical quality in the brain: ERP evidence for robust word learning from context. Dev Neuropsychol 35(4):376-403. doi:10.1080/ 87565641.2010.480915 
Gallés NS (2000) LEXESP: Léxico informatizado del español: Edicions Universitat Barcelona

Genovese CR, Lazar NA, Nichols T (2002) Thresholding of statistical maps in functional neuroimaging using the false discovery rate. Neuroimage 15(4):870-878. doi:10.1006/nimg.2001.1037

Groening K, Brodbeck V, Moeller F, Wolff S, van Baalen A, Michel CM, Siniatchkin M (2009) Combination of EEG-fMRI and EEG source analysis improves interpretation of spike-associated activation networks in paediatric pharmacoresistant focal epilepsies. Neuroimage 46(3):827-833. doi:10.1016/j.neuroimage. 2009.02.026

Groh-Bordin C, Zimmer HD, Ecker UK (2006) Has the butcher on the bus dyed his hair? When color changes modulate ERP correlates of familiarity and recollection. Neuroimage 32(4):1879-1890. doi:10.1016/j.neuroimage.2006.04.215

Herbster AN, Mintun M, Nebes R, Becker J (1997) Regional cerebral blood flow during word and nonword reading. Hum Brain Mapp 5(2):84-92. doi:10.1002/(SICI)1097-0193(1997)5:2<84:AIDBM2>3.0.CO;2-I

Hillyard SA, Teder-Sälejärvi WA, Münte TF (1998) Temporal dynamics of early perceptual processing. Curr Opin Neurobiol 8(2):202-210. doi:10.1016/S0959-4388(98)80141-4

Jasper HH (1958) The ten twenty electrode system of the international federation. Electroencephalogr Clin Neurophysiol 10:371-375

Joubert S, Beauregard M, Walter N, Bourgouin P, Beaudoin G, Leroux J-M, Lecours AR (2004) Neural correlates of lexical and sublexical processes in reading. Brain Lang 89(1):9-20. doi:10. 1016/S0093-934X(03)00403-6

Juphard A, Vidal JR, Perrone-Bertolotti M, Minotti L, Kahane P, Lachaux JP, Baciu M (2011) Direct evidence for two different neural mechanisms for reading familiar and unfamiliar words: an intra-cerebral EEG study. Front Hum Neurosci 5:101. doi:10. 3389/fnhum.2011.00101

Kim YY, Lee B, Shin YW, Kwon JS, Kim MS (2006) Activity of left inferior frontal gyrus related to word repetition effects: LORETA imaging with 128-channel EEG and individual MRI. Neuroimage 29(3):712-720. doi:10.1016/j.neuroimage.2005.08. 032

Kim MS, Jang KM, Che H, Kim DW, Im CH (2012) Electrophysiological correlates of object-repetition effects: sLORETA imaging with 64-channel EEG and individual MRI. BMC Neurosci 13(1):124. doi:10.1186/1471-2202-13-124

Koenig T, Melie-García L, Stein M, Strik W, Lehmann C (2008) Establishing correlations of scalp field maps with other experimental variables using covariance analysis and resampling methods. Clin Neurophysiol 119(6):1262-1270. doi:10.1016/j. clinph.2007.12.023

Kutas M, Federmeier KD (2011) Thirty years and counting: finding meaning in the N400 component of the event-related brain potential (ERP). Ann Rev Psychol 62:621-647. doi:10.1146/ annurev.psych.093008.131123

Kutas M, Hillyard SA (1980) Reading senseless sentences: brain potentials reflect semantic incongruity. Science 207(4427): 203-205. doi:10.1126/science.7350657

Laszlo S, Federmeier KD (2007) Better the DVL you know acronyms reveal the contribution of familiarity to single-word reading. Psychol Sci 18(2):122-126. doi:10.1111/j.1467-9280.2007.01859.x

Laszlo S, Stites M, Federmeier KD (2012) Won't get fooled again: an event-related potential study of task and repetition effects on the semantic processing of items without semantics. Language and cognitive processes 27(2):257-274. doi:10.1080/01690965.2011. 606667

Lehmann D, Skrandies W (1980) Reference-free identification of components of checkerboard-evoked multichannel potential fields. Electroencephalogr Clin Neurophysiol 48(6):609-621. doi:10.1016/0013-4694(80)90419-8
Levy J, Pernet C, Treserras S, Boulanouar K, Aubry F, Démonet JF, Celsis P (2009) Testing for the dual-route cascade reading model in the brain: an fMRI effective connectivity account of an efficient reading style. PLoS One 4(8):e6675. doi:10.1371/ journal.pone. 0006675

Maloney E, Risko EF, O'Malley S, Besner D (2009) Tracking the transition from sublexical to lexical processing: On the creation of orthographic and phonological lexical representations. Q J Exp Psychol 62(5):858-867. doi:10.1080/1747021080 2578385

Maris E, Oostenveld R (2007) Nonparametric statistical testing of EEG-and MEG-data. J Neurosci Methods 164(1):177-190. doi:10.1016/j.jneumeth.2007.03.024

McLaughlin J, Osterhout L, Kim A (2004) Neural correlates of second-language word learning: minimal instruction produces rapid change. Nat Neurosci 7(7):703-704. doi:10.1038/nn1264

Mechelli A, Humphreys GW, Mayall K, Olson A, Price CJ (2000) Differential effects of word length and visual contrast in the fusiform and lingual gyri during. Proc R Soc Lond B 267(1455): 1909-1913. doi:10.1098/rspb.2000.1229

Mestres-Missé A, Rodriguez-Fornells A, Münte TF (2007) Watching the brain during meaning acquisition. Cereb Cortex 17(8): 1858-1866. doi:10.1093/cercor/bhl094

Michel CM, Murray MM, Lantz G, Gonzalez S, Spinelli L, de Peralta RG (2004) EEG source imaging. Clin Neurophysiol 115(10): 2195-2222. doi:10.1016/j.clinph.2004.06.001

Mitchell PF, Andrews S, Ward PB (1993) An event-related potential study of semantic congruity and repetition in a sentence-reading task: effects of context change. Psychophysiology 30(5):496-509. doi:10.1111/j.1469-8986.1993.tb02073.x

Murray MM, Brunet D, Michel CM (2008) Topographic ERP analyses: a step-by-step tutorial review. Brain Topogr 20(4):249-264. doi:10.1007/s10548-008-0054-5

Newman SD, Twieg D (2001) Differences in auditory processing of words and pseudowords: an fMRI study. Hum Brain Mapp 14(1):39-47. doi:10.1002/hbm.1040

Nobre AC, Allison T, McCarthy G (1994) Word recognition in the human inferior temporal lobe. Nature 372(6503):260-263. doi: $10.1038 / 372260 \mathrm{a} 0$

Oldfield RC (1971) The assessment and analysis of handedness: the Edinburgh inventory. Neuropsychologia 9(1):97-113. doi:10. 1016/0028-3932(71)90067-4

Oostenveld R, Fries P, Maris E, Schoffelen J-M (2011) FieldTrip: open source software for advanced analysis of MEG, EEG, and invasive electrophysiological data. Comput Intell Neurosci 2011:1. doi:10.1155/2011/156869

Pedroni A, Langer N, Koenig T, Allemand M, Jäncke L (2011) Electroencephalographic topography measures of experienced utility. J Neurosci 31(29):10474-10480. doi:10.1523/JNEUR OSCI.5488-10.2011

Perfetti CA, Wlotko EW, Hart LA (2005) Word learning and individual differences in word learning reflected in event-related potentials. J Exp Psychol 31(6):1281. doi:10.1037/0278-7393. 31.6.1281

Price CJ (1998) The functional anatomy of word comprehension and production. Trends Cogn Sci 2(8):281-288. doi:10.1016/S13646613(98)01201-7

Renoult L, Wang X, Calcagno V, Prévost M, Debruille JB (2012) From N400 to N300: variations in the timing of semantic processing with repetition. NeuroImage 61(1):206-215. doi:10. 1016/j.neuroimage.2012.02.069

Ripamonti E, Aggujaro S, Molteni F, Zonca G, Frustaci M, Luzzatti C (2014) The anatomical foundations of acquired reading disorders: a neuropsychological verification of the dual-route model of reading. Brain Lang 134:44-67. doi:10.1016/j.bandl.2014.04. 001 
Rugg MD, Curran T (2007) Event-related potentials and recognition memory. Trends Cogn Sci 11(6):251-257. doi:10.1016/j.tics. 2007.04.004

Rugg MD, Yonelinas AP (2003) Human recognition memory: a cognitive neuroscience perspective. Trends Cogn Sci 7(7):313-319. doi:10.1016/S1364-6613(03)00131-1

Rumsey J, Horwitz B, Donohue B, Nace K, Maisog J, Andreason P (1997) Phonological and orthographic components of word recognition. A PET-rCBF study. Brain 120(5):739-759. doi:10. 1093/brain/120.5.739

Schneider W, Eschman A, Zuccolotto A (2002) E-Prime (Version 2.0). Computer software and manual. Psychology Software Tools Inc., Pittsburgh

Stein M, Dierks T, Brandeis D, Wirth M, Strik W, Koenig T (2006) Plasticity in the adult language system: a longitudinal electrophysiological study on second language learning. Neuroimage 33(2):774-783. doi:10.1016/j.neuroimage.2006.07.008

Taha H, Khateb A (2013) Resolving the orthographic ambiguity during visual word recognition in Arabic: an event-related potential investigation. Front Hum Neurosci 7:821. doi:10.3389/ fnhum.2013.00821

Talairach J, Tournoux P (1988) Co-planar stereotaxic atlas of the human brain: 3-Dimensional proportional system-an approach to cerebral imaging. Thieme Medical Publishers, New York
Van Atteveldt NM, Formisano E, Goebel R, Blomert L (2007) Topdown task effects overrule automatic multisensory responses to letter-sound pairs in auditory association cortex. Neuroimage 36(4):1345-1360

Van Petten C, Kutas M, Kluender R, Mitchiner M, McIsaac H (1991) Fractionating the word repetition effect with event-related potentials. J Cogn Neurosci 3(2):131-150. doi:10.1162/jocn. 1991.3.2.131

Van Strien JW, Hagenbeek RE, Stam CJ, Rombouts SA, Barkhof F (2005) Changes in brain electrical activity during extended continuous word recognition. Neuroimage 26(3):952-959. doi:10.1016/j.neuroimage.2005.03.003

Voss JL, Federmeier KD (2011) FN400 potentials are functionally identical to N400 potentials and reflect semantic processing during recognition testing. Psychophysiology 48(4):532-546. doi:10.1111/j.1469-8986.2010.01085.x

Wang Q, Yuan J (2008) N400 lexicality effect in highly blurred Chinese words: evidence for automatic processing. NeuroReport 19(2):173-178. doi:10.1097/WNR.0b013e3282f3e3f2

Ziegler JC, Besson M, Jacobs AM, Nazir TA, Carr TH (1997) Word, pseudoword, and nonword processing: A multitask comparison using event-related brain potentials. J Cogn Neurosci 9(6):758-775. doi:10.1162/jocn.1997.9.6.758 